\title{
The Cosmology of Edgar Allan Poe
}

\author{
Alberto Cappi ${ }^{1,2}$ \\ ${ }^{1}$ INAF - Osservatorio Astronomico di Bologna, \\ Via Ranzani 1, 40127 Bologna, Italy \\ email: alberto.cappi@oabo.inaf.it \\ ${ }^{2}$ Laboratoire Cassiopée, Observatoire de la Côte d'Azur, \\ BP4229, 06304 Nice Cedex 4, France
}

\begin{abstract}
Eureka is a "prose poem" published in 1848, where Edgar Allan Poe presents his original cosmology. While starting from metaphysical assumptions, Poe develops an evolving Newtonian model of the Universe which has many and non casual analogies with modern cosmology. Poe was well informed about astronomical and physical discoveries, and he was influenced by both contemporary science and ancient ideas. For these reasons, Eureka is a unique synthesis of metaphysics, art and science.
\end{abstract}

Keywords. cosmology, history and philosophy of astronomy, literature

\section{Introduction}

In the second half of the XVIIIth century there were interesting speculations concerning the nature and structure of the Universe, which combined the limited observations available at the time with Newtonian laws and metaphysical principles. In An Original Theory or New Hypothesis of the Universe, published in 1750, Thomas Wright identified the faint nebulae observed in the sky as distant stellar systems comparable to the Milky Way. We find the same idea† five years later, in the Natural History and Theory of Heavens (Naturgeschichte und Theorie des Himmels), written by the young Immanuel Kant. However, the first scientific cosmogony, restricted to the origin of the solar system, is the famous Nebular Hypothesis of Laplace, described in his Exposition du Système du Monde (1796). Laplace suggested that the gravitational contraction of a primordial, rotating nebula could have formed our planetary system and give origin to the observed regularities of planetary motions. In the meanwhile, the observations of William Herschel with his powerful telescopes seemed to confirm that all nebulae were distant stellar systems; later Herschel convinced himself of the existence of nebular matter in space, thus giving an observational support to the primordial nebula of Laplace.

These were the main scientific ideas concerning the structure of the Universe when, on February 3, 1848, at the Society Library of New York, Edgar Allan Poe read a lecture "On the Cosmogony of the Universe"; he revised the text and published it in June of the same year; the title of the book was: Eureka. A Prose Poem. see www. eapoe.org/works/ editions/eureka.htm

The content of Eureka is of course not entirely original, and there are many sources which inspired Poe: among them, we can mention von Humboldt's Kosmos, John Nichol's Views of the Architecture of the Heavens, John Herschel's A Treatise on Astronomy, David Brewster's The Martyrs of Science. However, the cosmology of Poe is original, and in its context old ideas are generalixed or assume a new meaning.

$\dagger$ Another theory on the structure of the Universe, with the identification of nebulae with stellar systems, is described by Johann Heinrich Lambert in his Cosmological Letters, published in 1761 . 
Eureka can be divided into three parts, which strongly differ in their style and content. The first part is a satirical discussion on the scientific method, where Poe wants to show that the knowledge of the Universe is possible, but it is necessary to abandon the old way of induction and search for consistency: "A perfect consistency can be nothing but an absolute truth". The second part presents Poe's cosmology with its metaphysical, physical and observational basis, and here we will focus on it. The third part is lyrical and spiritual, and constitutes an appropriate conclusion for a poem.

\section{The origin of the Universe}

The physical basis of Poe's universe are Newton's law of gravitation and the Nebular Hypothesis of Laplace. From the universality of gravitation, Poe infers that matter tends to come back to a primordial state of unity, corresponding to a primordial particle:

Let us now endeavor to conceive what Matter must be, when, or if, in its absolute extreme of Simplicity. Here the Reason flies at once to Imparticularity - to a particle - to one particle $[\ldots]$

Oneness, then, is all that I predicate of the originally created Matter; but I propose to show that this Oneness is a principle abundantly sufficient to account for the constitution, the existing phaenomena and the plainly inevitable annihilation of at least the material Universe.

How can we conciliate the existence of the stars we see in the sky with the original Oneness? Through the fragmentation of the primordial particle and the diffusion of atoms in space under the action of a repulsive force. The atoms were diffused in a large but non infinite, spherical region of space.

Simplicity also implies that the original distribution of atoms was homogeneous; according to Poe, this is confirmed by the fact that stars appear to be more or less uniformly distributed:

A very slight inspection of the Heavens assures us that the stars have a certain general uniformity, equability, or equidistance, of distribution through that region of space in which, collectively, and in a roughly globular form, they are situated [...]

Homogeneity is still the main postulate of modern cosmology, introduced in relativistic models by Einstein himself.

But Poe realizes that there is a problem:

I started, it will be remembered, with the idea of a generally uniform but particularly ununiform distribution of the atoms; -an idea, I repeat, which an inspection of the stars, as they exist, confirms. [...] Let me now describe the sole possible mode in which it is conceivable that matter could have been diffused through space, so as to fulfill the conditions at once of irradiation and of generally equable distribution.

In fact, diffusion into space would seem to imply increasing rarefaction at increasing distances from the center. How could the uniform distribution of matter be generated and preserved during the diffusion of atoms? Restated in modern terms, this means that homogeneity should be conserved during the expansion of the Universe: the only way to obtain this result, is that the recession velocity between galaxies must be proportional to their separation. From our point of view, of course, we observe all the other galaxies receding from us with a velocity proportional to their distance: this is what Hubble discovered in 1929. Poe could therefore predict the Hubble law, if he had concluded that the repulsive force should be proportional to the distance from the center of expansion, instead of claiming that it should be proportional to the square of the distance (for a metaphysical symmetry with the law of gravitation). Moreover, the repulsive force disappears and as a reaction attraction appears, a phenomenon Poe attributes to God's 
intervention. Notice that this repulsive force has a correspondence in modern cosmology: during the first instants after the Big Bang (Poe speaks of an "instantaneous flash"), inflation acts as a repulsive force which accelerates the expansion. Also inflation must come to an end, but this problem is solved in a more elegant way without divine intervention...

Poe discusses also the problem of structure formation from a homogeneous primordial state of the Universe (which is still a very active research field!).

How can we explain - the design of variety out of unity - diversity out of sameness - heterogeneity out of homogeneity - complexity out of simplicity -")? [...] The spatial distribution of atoms was uniform but they had a "difference of form at their dispersion, with particular inequidistance after it."

In modern cosmology, we attribute the origin of structure to primordial density fluctuations. Poe tries to produce these fluctuations by assuming that atoms have different shapes: this is why he claims that "We thus establish the Universe on a geometrical basis", which has no relation with Relativity but a lot in common with the ancient Greek atomism.

This primordial distribution of atoms represents a generalization of the primordial nebula of Laplace to the whole Universet, and the gravitational clustering of atoms gives origin to stars and clusters of stars, while the universe collapses towards its final end.

Let now examine the following quotations:

[... we could conceive the beginning of the universe in the form of a unique atom, the atomic weight of which is the total mass of the universe.

After the cosmogonies of Laplace and Kant, we have taken the habit of conceiving as a starting point of the evolution of the world a diffuse nebula filling the space and progressively condensing in partial nebulae, then in stars. [...] The world proceeded from a condensed to a diffused [state]. [...] The atom-universe broke off into fragments.

Cosmological theories aim to search for initial conditions ideally simple whence could result, through the natural play of known physical forces, the present world in all its complexity. It seems difficult to conceive conditions simpler than those which reigned when all matter was unified in an atomic nucleus. $\ddagger$

The above considerations seem an appropriate description of Poe's cosmology: nevertheless the author is not Poe, but Georges Lemaître, who is acknowledged to have made the first step towards the Big Bang theory. Lemaitre's suggestion of a primordial atom was based on the behaviour of relativistic cosmological models and the observations supporting the expansion of the Universe, while Poe had no available evidence at all, but their philosophical approach seems to be very similar (it is also worth mentioning that Lemaître, as Poe, believed that the Universe should be finite).

In the following section it will be clear that there are a lot of other analogies between Poe's Universe and modern cosmology. The technical reason of these analogies is that for a limited volume of space Newtonian cosmology is a good approximation of a homogeneous relativistic universe.

$\dagger$ As he had to explain the properties of the Solar System, Laplace assumed that the primordial nebula was rotating; while Poe assumes that the Universe does not rotate, and has only radial expansion and collapse, just as in our standard Friedmann-Lemaître models.

$\ddagger$ The three quotations come respectively from: The beginning of the world from the point of view of quantum theory, 1931, Nature, 127, 706; Expansion of the universe, 1931, MNRAS, 91, 483; L'hypothèse de l'atome primitif, 1944 (Neuchâtel: Editions du Griffon). 


\section{The physical cosmology of Poe}

After concluding that the Universe is evolving, and that it is now collapsing, Poe derives a number of interesting astrophysical consequences, showing that they are consistent with his cosmology.

First of all, Poe gives a detailed and accurate description of the formation of the Solar System according to the Nebular Cosmogony of Laplace. He tries to derive the consequences of Laplace's hypothesis on the birth and development of life.

However, the hypothesis of Laplace required the existence of nebular matter, which was called in question in 1846, when Lord Rosse (erroneously) claimed to have resolved the Orion Nebula into stars. This result suggested that all nebulae were stellar systems. Poe solves this problem in a simple and brilliant way, noting that if the process of star formation was accomplished in the past, now we can observe only stellar systems. This idea corresponds to our concept of a primordial epoch of galaxy formation, even if we know that star formation is continuing in galaxies (but at a much lower rate with respect to the past).

Poe stresses that the nebulae are stellar systems as the Milky Way:

The 'clusters' of which this Universal 'cluster of clusters' consists are merely what we have been in the practice of designating 'nebulae' and, of these 'nebulae', one is of paramount interest to mankind. I allude to the Galaxy, or Milky Way.

$[\ldots]$

We have no reason to suppose the Milky Way really more extensive than the least of these 'nebulae'.

Such systems are at distances of millions of light-years, according to the estimates of William Herschel. Unfortunately this view of the Universe was abandoned in the second half of the XIXth century. Poe insists that the cluster of clusters must be finite, using what we call now the Olbers' paradox: the night sky cannot be dark in an eternal Universe with a homogeneous distribution of stars. The reason is that the flux coming from a single star decreases with the square of its distance, while the number of stars in shells of equal width and concentric to the earth increases with the square of their distance. The result is that the total flux coming from each shell is constant, independent of its distance. Therefore if we have infinite shells, we have an infinite flux. Even taking into account that foreground stars stop the light of background stars, the whole sky should be as luminous as the Sun. One possible and correct solution is that the number of stars and stellar systems is finite, as in Poe's Universe. However, Poe admits that there is another possibility to solve the paradox, if we believe that the Universe is infinite:

Were the succession of stars endless, then the background of the sky would present us an uniform luminosity, like that displayed by the Galaxy - since there could be absolutely no point, in all that background, at which would not exist a star. The only mode, therefore, in which, under such a state of affairs, we could comprehend the voids which our telescopes find in innumerable directions, would be by supposing the distance of the invisible background so immense that no ray from it has yet been able to reach us at all.

This represents, as noted by Harrison (1987), the first correct solution of the Olbers' paradox for an infinite universe. This solution is based on the observation that light has a finite speed: therefore we cannot see a star if its light cannot reach us in less than the age of the Universe. Even if the Universe is infinite, the observable Universe is not, of course if we assume that the Universe has a finite age.

It seems to me that Poe was inspired by von Humboldt's discussion of the Olbers' paradox in Kosmos, where von Humboldt mentions the regions of the sky without stars 
discovered by William Herschel (these "voids" were indeed regions of our Galaxy obscured by concentrations of gas and dust). Perhaps, von Humboldt suggests,

[...] other stars could also be there, but our instruments are not able to reach them.

While this could be a reasonable hypothesis for explaining the voids, it is certainly a wrong solution of the Olbers' paradox. Poe follows von Humboldt and relates the observation of voids to the Olbers' paradox, but with a crucial difference: it is the light of distant stars which has not yet been able to reach us. Poe implicitly assumes that the speed of light is finite, and that the Universe has a finite age.

The observation of large voids is related to another interesting and old problem of cosmology: the vastness of the Universe. With his evolving universe, Poe finds a new and original solution:

A rational cause for the phaenomenon, I maintain that Astronomy has palpably failed to assign: - but the considerations through which, in this Essay, we have proceeded step by step, enable us clearly and immediately to perceive that Space and Duration are One. That the Universe of Stars might endure throughout an aera at all commensurate with the grandeur of its component material portions and with the high majesty of its spiritual purposes, it was necessary that the original atomic diffusion be made to so inconceivable an extent as to be only not infinite. It was required, in a word, that the stars should be gathered into visibility from invisible nebulosity - proceed from visibility to consolidationand so grow grey in giving birth and death to unspeakably numerous and complex variations of vitalic development: -it was required that the stars should do all this -should have time thoroughly to accomplish all these Divine purposes-during the period in which all things were effecting their return into Unity with a velocity accumulating in the inverse proportion of the squares of the distances at which lay the inevitable End.

By claiming that Space and Duration are One, Poe was not anticipating Einstein's Relativity or Minkowski's concept of space-time, but he had discovered a fundamental property of the evolving Universe. The meaning of Poe's discovery becomes clear when reading what the cosmologist John Barrow has written 140 years later:

[...] This state of expansion means that the size of the Universe is inextricably entwined with its age. The reason that the Visible Universe is more than 13 billion light-years in size today is that it is more than 13 billion years old. A Universe that contained just one galaxy like our own Milky Way, with its 100 billion stars, each perhaps surrounded by planetary systems, might seem a reasonable economy if one were in the universal construction business. But such a universe, with more than a 100 billion fewer galaxies than our own, could have expanded for little more than a few months. It could have produced neither stars nor biological elements. It could contain no astronomers $\dagger$.

In the evolving Newtonian Universe of Poe, as well as in the modern relativistic models, the age and size of the Universe are related $\ddagger$. Moreover, our existence implies that the Universe must be sufficiently old, therefore its size must be correspondingly large. This is an application of what we call the Anthropic Principle (Barrow \& Tipler 1986).

\section{Many Universes}

The conclusion of Eureka has a lyrical tone and is quite different in content from the rest of the work. In this part, Poe extends his theory in a surprising way.

He claims that after the final annihilation of the Universe, God will create another

$\dagger$ Barrow J.D., 1988, The World within the World, (Oxford: Clarendon Press), p. 354

$\ddagger$ The only difference, irrelevant in this case, is that Poe believed we are living during the collapse of the Universe. 
Universe, with another cycle of diffusion and collapse, and so on. He claims also that there is a "limitless" number of universes in space, and notes that different universes do not interact between them, as they have different origins (thus avoiding all the problems posed by an infinite Universe).

Why does Poe extend his theory? One reason, I suspect, is symmetry (and for Poe, "symmetry and consistency are convertible terms"). In Newtonian cosmology, a material Universe limited in space and time defines a privileged region of space and a privileged interval of time. Unsatisfied by this situation, Poe could have conceived his generalization. However, I have recently found clear evidence that he was inspired in his conception both of gravity and of other, non interacting universes by old Greek ideas described in two works of Plutarch, De Facie quae in Orbe Lunae apparet, explicitly quoted by Poe in one of his tales in 1845, and De Defectu Oraculorum. This is confirmed by another, astonishing aspect of Poe's cosmology: when claiming the existence of other Universes, he writes:

Each [Universe] exists, apart and independently, in the bosom of its proper and particular God.

This implies an infinite number of gods! Such a surprising polytheism could be a consequence of the principle of symmetry applied to the spiritual Universe, but it might also reflect a debate on the existence of other gods and universes in Plutarch's De Defectu Oraculorum. I will describe in detail this question in another paper (Cappi 2009).

We have seen that Poe starts with one Universe, and ends assuming that there are other Universes; the Big Bang theory was initially considered the description of the Universe: now it is considered as an incomplete theory and among cosmologists it is very popular the concept of the "Multiverse", i.e. the existence of other universes, in order to justify the incredible fine tuning of fundamental constants, on which our existence depends. In fact, this fine tuning would not be surprising if physical constants could have different values in different universes; in most universes intelligent life will not be possible, while our existence implies that in our Universe fundamental constants must have particular values (an application of the Anthropic Principle).

The final part of Eureka has also startling spiritual implications. Poe believes that God himself is diffused into our souls, and that "The Universe is but his present expansive existence". When the material Universe will reach its final Unity and will annihilate, our souls will come to Unity with God. Poe thinks that our identity will be lost, but there is a consolation:

That God may be all in all, each must become God.

The conclusion of Eureka cannot be judged in the same way as the previous parts; it is the surprising and magnificent ending of a poem. Scientific cosmology cannot describe the "spiritual" nature of the Universe, while art has not such limits. Poe has left us, one year before his premature death, a unique work, in the tradition of the scientific poems of Empedocles and Lucretius: a fascinating and inspiring synthesis of art, metaphysics and science midway between ancient ideas and contemporary cosmology.

\section{References}

Barrow, J. D. \& Tipler, F. J. 1986, The Anthropic Cosmological Principle (Oxford: Oxford University Press)

Cappi, A. 1994, Quarterly Journal of the R.A.S., 35, 177

Cappi, A. 2004, in Cosmology through Time: Ancient and Modern Cosmologies in the Mediterranean Area, S. Colafrancesco and G. Giommi (eds) (Milano: Mimesis), p. 239

Harrison, E. 1987, Darkness at Night. A Riddle of the Universe (Cambridge: Harvard University Press) 